\title{
Effect of Slowly Forced Expiration on Abdominal Muscle Activity During Cross Knee Curl-Up Exercise
}

\author{
Tae-lim Yoon ${ }^{1,2}$, MA, PT, Ki-song $\mathrm{Kim}^{3}$, PhD, PT \\ ${ }^{1}$ Applied Kinesiology and Ergonomic Technology Laboratory \\ ${ }^{2}$ Dept. of Physical Therapy, The Graduate School, Yonsei University \\ ${ }^{3}$ Dept. of Physical Therapy, College of Natural Science, Hoseo University
}

\begin{abstract}
Cross knee curl-up is an ideal variation of abdominal curl up exercise to strengthen abdominal musculature without excessive lumbar flexion which can increase the loads on the disc and ligaments. In addition, slowly forced expiration can facilitate the activation of the abdominal musculature. The purpose of this study was to determine the effects of slowly forced expiration on activity of abdominal muscles, such as rectus abdominis (RA), external oblique (EO), and transverse abdominis/internal oblique ( $\operatorname{Tr} \mathrm{A} / \mathrm{IO})$, while cross knee curl-up. Eleven young and healthy subjects (6 males and 5 females) participated. All subjects performed the cross knee curl-up slowly forced expiration and natural breathing. Paired t-test was performed in normalized electromyogram (EMG) muscle activity of the bilateral RA, EO, and TrA/IO to compare the differences between the cross curl-up with slowly forced expiration and natural breathing. Statistical significance was set at .05 . There were no significant differences in normalized EMG muscle activity of the bilateral RA, EO, and TrA/IO between the cross curl-up with slowly forced expiration and natural breathing. The finding of this study designates that slowly forced expiration does not induce increasing activity of abdominal muscle in cross knee curl-up; hence, learning step of breathing control might not be necessary to strengthen abdominal muscle in cross knee curl-up.
\end{abstract}

Key Words: Breathing; Respiration; Stabilization exercise.

\section{Introduction}

Abdominal curl-up exercise can be recommended for strengthening abdominal muscles and stabilizing lumbar area as a therapeutic exercise in rehabilitation program (Axler and McGill, 1997; McGill and Karpowicz, 2009). Also, abdominal curl-up exercise can be selected as an aerobic exercise for healthy people to strengthen abdominal muscles by health instructors in pilates, athletic and rehabilitation program (Critchley et al, 2011; Hides et al, 2011). However, the abdominal curl-up exercise also may activate the hip flexor and lumbar paraspinal muscle, a result that may not be beneficial for low back pain patient. For example, high levels activation of the hip flexor and lumbar paraspinal muscles have a ten- dency to rotate the pelvis anteriorly, increase lumbar lordosis and increase lumbar segmental compression, thus increasing the risk of low back pain (Ralston et al, 1990).

A previous study reported that cross knee curl-up is an ideal variation of abdominal curl up exercise to strengthen abdominal musculature without lumbar flexion which can increase the loads on the disc and ligaments (McGill, 1998; McGill, 2007). The previous researcher advocated that the cross knee curl-up can be applied to initial physical therapy for the patients with low back pain to keep neutral spine posture without pain.

Expiration is considered a passive process during quiet breathing, but with an increased breathing effort, forced expiration can facilitate the activation of

Corresponding author: Ki-song Kim kskim68@hoseo.edu 
the abdominal musculature (Simpson, 1983). The pressure of intra-abdominal is increased and the diaphragm is moved upwards for forced expiration while the abdominal musculature contracts (Campbell and Green, 1953; Woorons et al, 2007). Therefore, forced expiration can be applied to abdominal curl-up exercise and the other core stabilizing exercise (Cho et al, 2013; Kim and Lee, 2013; Lee et al, 2013). Particularly, combined task of the cross knee curl-up and forced expiration would be beneficial with increasing abdominal muscle activity while as maintaining of neural spine during core stabilizing exercise. To the best of knowledge, this is the original study to examine the effects of slowly forced expiration (Woorons et $\mathrm{al}, 2007$ ) on activity of $\mathrm{ab}^{-}$ dominal muscles such as rectus abdominis (RA), external oblique (EO), and transverse abdominis/internal oblique (TrA/IO). These muscles are defined as major expiratory muscles (Neumann, 2002).

Hence, the purpose of this study was to determine the effects of slowly forced expiration on activity of abdominal muscles such as RA, EO, and $\operatorname{TrA} / \mathrm{IO}$ during performing of cross knee curl-up. The research hypothesis was that abdominal muscle activity will be differences of abdominal muscles activity in cross knee curl-up with slowly forced expiration compared with natural breathing.

\section{Methods}

\section{Subjects}

Eleven healthy subjects participated in this re $^{-}$ search (Table 1). Exclusion criteria were history of abdominal or lower back pain within six weeks of start of the study, past history of respiratory diseases, or inability to correctly perform the cross knee curl-up in a pain-free manner (Roussel et al, 2009; Youdas et al, 2008). In addition, The dominance of leg was determined based on asking the subject to kick a soccer ball (Jacobs et al, 2005; Sung, 2013). All participants participate in this study were right-leg dominant. All subjects provided written informed consent in agreement with the procedures of the Yonsei University Wonju Institutional Review Board.

\section{Instrumentation}

Surface electromyogram (EMG) was used to measure activity of abdominal muscles. The EMG data were collected bilaterally from the RA: electrode positioned $2 \mathrm{~cm}$ lateral from the midline of the umbilicus, EO: electrode positioned halfway between the most inferior point of the costal margin of the ribs and the anterior superior iliac spine (ASIS) and angled toward the pubic symphysis in a parallel direction to the fibers of the EO, and $\operatorname{Tr} \mathrm{A} / \mathrm{IO}$ : electrode positioned in a horizontal direction within a triangle consisting of a medial border made up of a line from the umbilicus to the pubic symphysis, an inferior border made up of a line from the ASIS to pubis symphysis, and a superior border made up of a line from ASIS to ASIS (Youdas et al, 2008). A previous study identified that the EMG signal gained from an electrode inferior to the ASIS represents the combined activity of the IO and TrA (Marshall and Murphy, 2003). Therefore, the EMG signal from TrA/IO electrode was considered as a combined activity of TrA and IO muscle. Before positioning the electrodes over each muscle, the skin of electrode sites was shaved, swabbed and sanded with alcohols-oaked cotton to decrease skin resistance. Disposable $\mathrm{Ag} / \mathrm{AgCl}$ surface electrodes were located parallel to the muscle fibers with $2 \mathrm{~cm}$ center-to-center arrangement via using the Tele-Myo 2400T EMG device with a wireless telemetry system

Table 1. General characteristics of subjects $\quad(\mathrm{N}=11)$

\begin{tabular}{cc}
\hline \hline Parameters & Mean $\pm \mathrm{SD}^{\mathrm{a}}$ \\
\hline Age (year) & $24.0 \pm 1.2$ \\
Height $(\mathrm{cm})$ & $160.0 \pm 7.3$ \\
Weight $(\mathrm{kg})$ & $55.0 \pm 10.6$ \\
$\mathrm{BMI}^{\mathrm{b}}\left(\mathrm{kg} / \mathrm{m}^{2}\right)$ & $21.5 \pm 2.3$ \\
\hline
\end{tabular}

${ }^{a}$ mean \pm standard deviation, body mass index. 
(Noraxon Inc., Scottsdale, AZ, USA).

On completing the electrode attachment, EMG data were recorded at the $1000 \mathrm{~Hz}$ sampling rate and analyzed with Myo-Research Master Edition 1.06 XP software (Noraxon Inc., Scottsdale, AZ, USA). The raw signal was filtered using a digital band-pass filter (Lancosh FIR) between 20 and $400 \mathrm{~Hz}$ to eliminate movement artifacts, and a $60 \mathrm{~Hz}$ notch filter was used to diminish electrical noise. Root-mean-square (RMS) values were considered with a moving window of 50 ms. For normalization, a 5-second maximal voluntary isometric contraction (MVIC) was measured 3 times per the abdominal muscles to determine a basis for EMG signal amplitude normalization.

The protocol for MVIC testing was adopted from a previous study (Escamilla et al, 2006). For the RA, the subject was positioned supine in a hook-lying position with the feet supported and the thoracolumbar spine maximally flexed (curl-up position) on the table. Then, a researcher applied manual $\mathrm{re}^{-}$ sistance to the subject's shoulders in the direction of trunk extension. For the EO, the subject was positioned supine in a hook-lying position with feet flat on the table. The trunk was maximally flexed and rotated to the left, with applying manual resistance at the shoulders in the direction of trunk extension and right rotation. For the TrA/IO muscle, the subject was positioned supine in a hook-lying position with the trunk flexed and maximally rotated to the right. A researcher applied manual resistance at the shoulders in the direction of trunk extension and left rotation. We used central 3 seconds of collected EMG data to decide the mean amplitude of MVIC. The normalized activity of each muscle was presented as a percentage of MVIC.

\section{Procedures}

All subjects had a familiarization session to understand how to perform the cross knee curl-up. Then, the subject learned how to correctly perform the cross knee curl-up with slowly forced expiration for 7 seconds. Once the subject correctly performed the cross knee curl-up for 7 seconds, data collection session were started. At the beginning, cross knee curl-up with natural breathing were measured twice with the dominant leg straight. After 30 min of rest time, cross knee curl-up with slowly forced expiration were measured (Wojtys et al, 1996). During data collection, the researcher monitored the subject for appropriate slowly forced expiration by listening for airflow sounds and observing mouth and lip motions. If a subject was assumed of not achieving the appropriate breath condition, the data of trial were not collected.

\section{The cross knee curl-up}

McGill (1998) suggested that the cross knee curl-up would be recommended in the early stages of training or rehabilitation due to helping stabilize the pelvis and support the neutral spine. Subjects were positioned in a supine position by an investigator and how to perform the cross knee curl-up. Each subject was asked to place hands under lumbar spine to preserve a neutral lumbar spine. Right knee was straight while left knee were 90 flexed. Subjects elevated the trunk by lifting the head and shoulders such that the scapulae were lifted above the table. We installed a target bar that could be located to the chest at the point when the scapulae came off the table (Figure 1). This was to guarantee that both scapulae were constantly elevated above the ta ${ }^{-}$

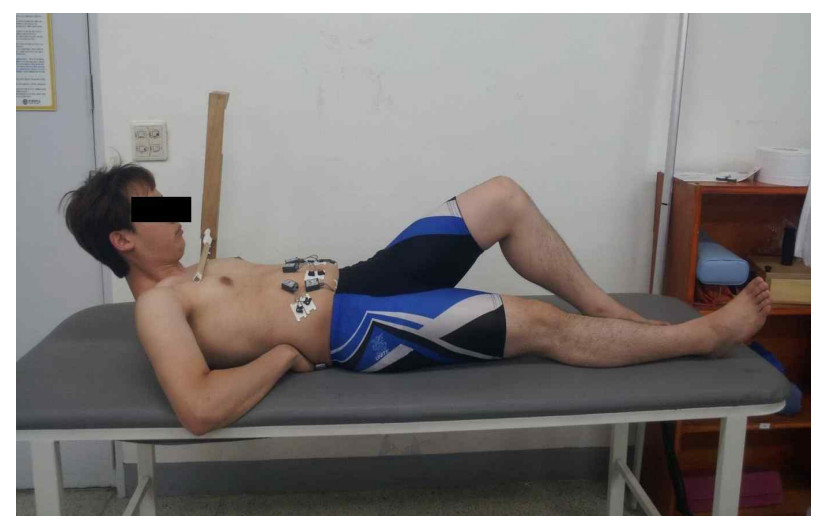

Figure 1. The cross knee curl-up with dominant leg straight. 
ble for each abdominal curl-up exercise. The subject was asked to touch the target bar during the cross knee curl-up. The subject was requested to hold the position isometrically for 7 seconds without hold of breathing to activate abdominal muscles. Then the subject returned to the starting position.

\section{Slowly forced expiration and natural breathing}

All subjects were supine and maintained the posture during this study. The subject was instructed to slowly inhale as deeply as possible and then to $\mathrm{ex}^{-}$ hale as forcefully and completely with compensation movement of the trunk (Lee et al, 2013). For natural breathing, there was no specific instruction of breathing pattern to all subjects. A researcher visually observed the subjects breathing pattern during data collection to ensure the slowly forced expiration and natural breathing.

\section{Statistical analysis}

Kolmogorov-Smirnov Z-tests were performed to assess the normality of distribution. Statistical significance was set at .05. Paired t-test was performed in normalized EMG muscle activity of the bilateral RA, EO and TrA/IO muscles to compare the differences between the cross curl-up with slowly forced expiration and natural breathing. Statistical analysis was calculated with SPSS ver. 18.0 software (SPSS, Inc., Chicago, IL, USA).

\section{Results}

All of the continuous variables were found to approximate a normal distribution (Kolmogorov-Smirnov $Z$-test, $\mathrm{p}>$.05). There were no significant differences in normalized EMG muscle activity (right RA: $\mathrm{t}=1.398, \mathrm{p}=.192$; left $\mathrm{RA}: \mathrm{t}=.978, \mathrm{p}=.351$; right $\mathrm{EO}$ : $\mathrm{t}=.920, \mathrm{p}=.379$; left EO: $\mathrm{t}=.472, \mathrm{p}=.647$; right $\operatorname{TrA} / \mathrm{IO}$ : $\mathrm{t}=1.588, \mathrm{p}=.143$; left $\operatorname{TrA} / \mathrm{IO}: \mathrm{t}=1.621, \mathrm{p}=.136)$ between the cross curl-up with slowly forced expiration and natural breathing (Figure 2).

\section{Discussion}

The purpose of this study was to investigate the

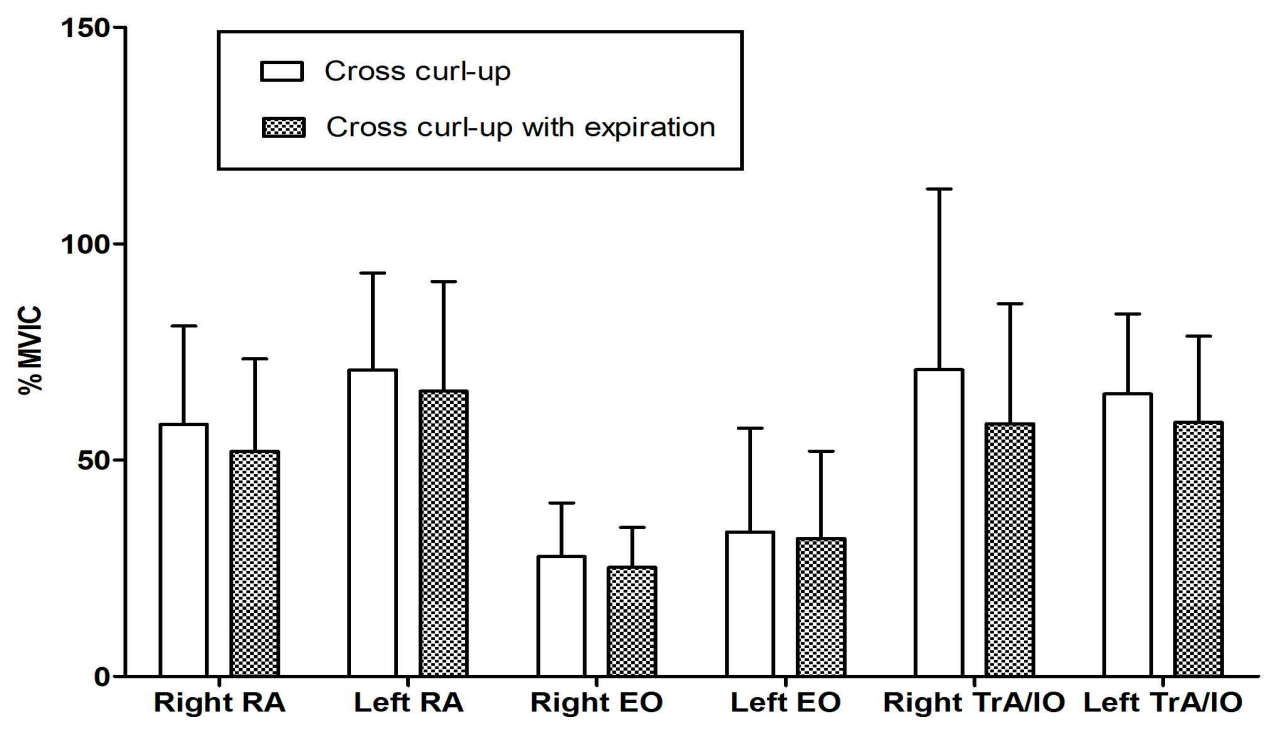

Figure 2. Normalized electromyogram muscle activity of the bilateral RA, EO, and TrA/IO muscles between the cross curl-up with slowly forced expiration and natural breathing (RA: rectus abdominis, EO: external oblique, TrA/IO: transverse abdominis/internal oblique). 
effects of slowly forced expiration on muscle activity of $\mathrm{RA}, \mathrm{EO}$ and $\operatorname{Tr} \mathrm{A} / \mathrm{IO}$ during favorable cross knee curl-up in healthy subjects. The research hypothesis was that abdominal muscle activity will be different in cross knee curl-up with slowly forced expiration compared with natural breathing. The results of this study showed that there were no significant differences in RA, EO, and $\operatorname{TrA} / \mathrm{IO}$ between cross knee curl-up with slowly forced expiration and natural breathing. Thus the findings of this study did not support research hypothesis.

There were no significant differences in RA, EO, and $\operatorname{Tr} \mathrm{A} / \mathrm{IO}$ between cross knee curl-up with slowly forced expiration and natural breathing. We supposed that there could be summative effect of dual task performance (Silsupadol et al, 2009) of slowly forced expiration and cross knee curl-up. A previous study presented that slow expiration would increase the TrA/IO muscle activity (about 35\%) in healthy subjects compared with those in abdominal curl up with quiet inspiration during traditional curl-up exercise (Yoon et al, 2013). However, the slowly forced $\mathrm{ex}^{-}$ piration could not effect on abdominal muscles during one task of cross knee curl-up in our study. This result might be explained with difficulty of dual task performance due to the lack of sufficient familiarization for the participant to controlling their breathing pattern during exercise. Previous studies reported that dual task showed greater task difficulty and required more practice compared with single task (Negahban et al, 2013; Vaportzis et al, 2013; Venema et al, 2013). In breathing task, dual task did elicit significantly greater elevations in heart rate, respiratory rate, and minute ventilation than single task (Webb et al, 2010). Due to the difficulty of modifying breathing pattern in relatively short time, the abdominal muscles were more focused in the cross knee curl-up than breathing task. If we used fast forced expiration than slow forced expiration, the muscular activity of the abdominal muscles may have been different.

The other possible explanation might be that slowly forced expiration is physiological phenomena which would not counted as a single task, therefore, cross knee curl-up could combined with this physiological expiration could not generate any difference in muscle activity for the health subjects.

Although there were no significant differences in muscle activity between cross knee curl-up with slowly forced expiration and natural breathing, there were slightly decreased activation of all muscles during cross knee curl-up with slowly forced expiration. We assumed that maintaining of neutral spine by placing hand under lumbar spine would change the muscle-tension relationship and influence on activation of muscles. Maintaining of neutral spine during core stabilization exercise is important because repetitively unwanted back flexion during abdominal curl-up could load mechanical stress on lumbar disc and ligaments which might worse mechanical receptor in patients with low back pain in the phase of rehabilitation program (Panjabi, 1992). In addition, maintaining of neutral spine has shown the better co-activation of the TrA (Sapsford et al, 2010). Therefore, maintaining of neutral spine and controlling of breathing pattern during curl-up $\mathrm{ex}^{-}$ ercise should be considered for individuals with sub-acute or chronic LBP in clinic.

There are several limitations. First, healthy subject participated in this study limiting external validity for the patients with low back pain. Second, there is no data of participant's exertion scale for control slowly forced expiration during cross knee curl-up. Third, there is no clear evidence of supporting the neural spine curve by placing hands under lumbar spine. Fourth, internal intercostal muscle is other major muscle of the forced expiration, there is no data of internal intercostal activity for estimation of compensatory using instead of abdominal muscle during slowly forced expiration in this experimental exercise. The collection of activity in internal intercostal muscle and participant's exertion scale for measuring of the difficulty in performance will add internal validity of the future study. 


\section{Conclusion}

This study compared the effects of slowly forced expiration and natural breathing on muscle activity of RA, EO, and $\mathrm{Tr} \mathrm{A} / \mathrm{IO}$ during cross knee curl-up. The finding of this study designates that slowly forced expiration does not induce increasing activity of abdominal muscle; hence, learning step of breathing control might not be necessary to strengthen $\mathrm{ab}^{-}$ dominal muscle in cross knee curl-up.

\section{References}

Axler CT, McGill SM. Low back loads over a variety of abdominal exercises: Searching for the safest abdominal challenge. Med Sci Sports Exerc. 1997;29(6):804-811.

Campbell EJ, Green JH. The expiratory function of the abdominal muscles in man: An electromyographic study. J Physiol. 1953;120(3):409-418.

Critchley DJ, Pierson Z, Battersby G. Effect of pilates mat exercises and conventional exercise programmes on transversus abdominis and obliquus internus abdominis activity: Pilot randomised trial. Man Ther. 2011;16(2):183-189.

Cho SH, Kim KH, Baek IH, et al. Comparison of contraction rates of abdominal muscles of chronic low back pain patients in different postures. J Phys Ther Sci. 2013;25(8):907-909.

Escamilla RF, McTaggart MS, Fricklas EJ, et al. An electromyographic analysis of commercial and common abdominal exercises: Implications for rehabilitation and training. J Orthop Sports Phys Ther. 2006;36(2):45-57.

Hides JA, Lambrecht G, Richardson CA, et al. The effects of rehabilitation on the muscles of the trunk following prolonged bed rest. Europ Spine J. 2011;20(5):808-818.

Jacobs C, Uhl TL, Seeley M, et al. Strength and fatigability of the dominant and nondominant hip abductors. J Athl Train. 2005;40(3):203-206.
Lee SK, Park JW, Kim KH, et al. An analysis of the thickness of abdominal muscles during forceful expiration and pulmonary function in teenage smokers and nonsmokers. J Phys Ther Sci. 2013;25(7):789-791.

Kim E, Lee $\mathrm{H}$. The effects of deep abdominal muscle strengthening exercises on respiratory function and lumbar stability. J Phys Ther Sci. 2013;25(6):663-665.

Marshall P, Murphy B. The validity and reliability of surface EMG to assess the neuromuscular $\mathrm{re}^{-}$ sponse of the abdominal muscles to rapid limb movement. J Electromyogr Kinesiol. 2003;13(5): 477-489.

McGill SM, Karpowicz A. Exercises for spine stabilization: Motion/motor patterns, stability progressions, and clinical technique. Arch Phys Med Rehabil. 2009;90(1):118-126.

McGill SM. Lumbar spine stability: Mechanism of injury and restabilization. In: Liebenson $\mathrm{C}$ ed. Rehabilitation of the Spine. 2nd ed. Philadelphia, Lippincott Williams \& Wilkins, 2007:106-107.

McGill SM. Low back exercises: Evidence for improving exercise regimens. Phys Ther. 1998;78(7):754-765.

Negahban H, Aryan N, Mazaheri M, et al. Effect of expertise in shooting and taekwondo on bipedal and unipedal postural control isolated or concurrent with a reaction-time task. Gait and Posture. 2013;38(2):226-230.

Neumann D. Kinesiology of the Musculoskeletal System: Foundations for physical rehabilitation. 1st ed. St. Louis, MO, Mosby, 2002:454-464.

Panjabi MM. The stabilizing system of the spine. Part I. Function, dysfunction, adaptation, and enhancement. J Spinal Disord. 1992;5(4):383-389.

Ralston SH, Urquhart GD, Brzeski M, et al. Prevalence of vertebral compression fractures due to osteoporosis in ankylosing spondylitis. BMJ. 1990;300(6724):563-565.

Roussel N, Nijs J, Truijen S, et al. Altered breathing patterns during lumbopelvic motor control tests 
in chronic low back pain: A case-control study. Eur Spine J. 2009;18(7):1066-1073.

Sapsford R, Hodges P, Smith M. Systematic review: Abdominal or pelvic floor muscle training. Neurourol Urodyn. 2010;29(5):800-801.

Silsupadol P, Lugade V, Shumway-Cook A, et al. Training-related changes in dual-task walking performance of elderly persons with balance impairment: A double-blind, randomized controlled trial. Gait Posture. 2009;29(4):634-639.

Simpson LS. Effect of increased abdominal muscle strength on forced vital capacity and forced expiratory volume. Phys Ther. 1983;63(3):334-337.

Sung PS. A compensation of angular displacements of the hip joints and lumbosacral spine between subjects with and without idiopathic low back pain during squatting. J Electromyogr Kinesiol. 2013;23(3):741-745.

Vaportzis E, Georgiou-Karistianis N, Stout JC. Dual task performance in normal aging: A comparison of choice reaction time tasks. PLoS One. 2013;8(3).

Venema DM, Bartels E, Siu KC. Tasks matter: A cross $^{-}$sectional study of the relationship of $\mathrm{cog}^{-}$ nition and dual-task performance in older adults. J Geriatr Phys Ther. 2013;36(3):115-122.

Webb HE, McMinn DR, Garten RS, et al. Cardiorespiratory responses of fire fighters to a computerized fire strategies and tactics drill during physical activity. Appl Ergon. 2010;41(3): 376-381.

Wojtys EM, Kothari SU, Huston LJ. Anterior cruciate ligament functional brace use in sports. Am J Sports Med. 1996;24(4):539-546.

Woorons X, Mollard P, Pichon A, et al. Prolonged expiration down to residual volume leads to $\mathrm{se}^{-}$ vere arterial hypoxemia in athletes during submaximal exercise. Respir Physiol \& Neurobiol. 2007;158(1):75-82.

Yoon TL, Kim KS, Cynn HS. Slow expiration reduces sternocleidomastoid activity and increases transversus abdominis and internal oblique muscle activity during abdominal curl-up. J Electromyogr Kinesiol. 2013. [Epub ahead of print]

Youdas JW, Guck BR, Hebrink RC, et al. An electromyographic analysis of the ab-slide exercise, abdominal crunch, supine double leg thrust, and side bridge in healthy young adults: Implications for rehabilitation professionals. J Strength Cond Res. 2008;22(6):1939-1946.

This article was received January 13, 2014, was reviewed January 13, 2014, and was accepted February 6, 2014. 